\title{
REVIEW
}

\section{Night-time symptoms: a forgotten dimension of COPD}

\author{
A. Agusti*,\#, J. Hedner", J.M. Marin ${ }^{+}$, F. Barbé ${ }^{\S, f}$, M. Cazzola** and S. Rennard ${ }^{\# \#}$
}

ABSTRACT: Sleep quality is often poor in patients with chronic obstructive pulmonary disease (COPD), but these night-time symptoms are frequently unnoticed by physicians and/or not reported by patients themselves. Therefore, the prevalence and clinical impact of sleep disturbances and night-time symptoms in COPD is not well understood and has not been a clinical focus to date. To address this gap, an expert panel meeting was convened in Barcelona, Spain, in March 2011 to discuss the aetiology, evolution, burden, long-term clinical consequences and optimal management of night-time symptoms in COPD. The term "night-time symptoms" in COPD has not been distinctly defined in an objective sense but epidemiological data suggests that the prevalence of nocturnal symptoms and symptomatic sleep disturbance may exceed $75 \%$ in patients with COPD. The panel concluded that night-time symptoms in COPD are prevalent and bothersome; that their cause(s) are multiple and include demographic factors, such as age and obesity, pharmacotherapy, disease-specific symptoms and the presence of comorbid sleep disorders, and other medical conditions; and that potential long-term consequences can include lung function changes, increased exacerbation frequency, emergence or worsening of cardiovascular disease, cognitive effects, depression, impaired quality of life and increased mortality. To date, few interventional studies have investigated them, but emerging data suggest that bronchodilator therapy can improve them if deployed appropriately. In summary, night-time symptoms in COPD warrant further clinical investigation with validated tools.

\section{KEYWORDS: Burden, chronic bronchitis, emphysema, pharmacotherapy, sleep disturbance}

hronic obstructive pulmonary disease (COPD) is a complex heterogeneous disease with numerous pulmonary and extra-pulmonary manifestations [1]. Even though patients with COPD often report poor sleep quality [2, 3], night-time symptoms and sleep disturbances in COPD are generally not considered in their clinical management. For instance, the most recent Global Initiative for Chronic Obstructive Lung Disease guidelines [4] do not mention sleep disturbance or night-time symptoms as targets for therapeutic intervention, and no or very limited specific guidance is offered on appropriate management strategies or pharmacological interventions for patients with COPD who report disrupted sleep. Paradoxically, the presence of sleep disorders is acknowledged as a potential comorbidity in COPD patients, and guidelines recommend asking patients about sleep disruption due to breathlessness or other symptoms during follow-up visits [4]. In fact, the nature and aetiology of disturbed sleep in COPD has been poorly characterised so far, and the long-term clinical consequences of sleep disturbance in COPD have yet to be explored.

To address these issues, an expert panel meeting was convened on March 28, 2011 in Barcelona, Spain with the goal of discussing the aetiology, evolution, burden and management of night-time symptoms in COPD, as well as to explore current knowledge on how they impact long-term outcomes in these patients. Although nocturnal respiratory symptoms, as well as symptomatic sleep disturbance, appear to be common in patients with COPD the term "night-time symptoms" has not been distinctly defined in an objective sense. Therefore, the panel also considered lessons from other disease areas, including asthma and sleep apnoea, and considered how research and understanding of night-time symptoms in these other diseases might impact our understanding and management of nighttime symptoms in COPD. This paper summarises

\section{AFFILIATIONS}

*Thorax Institute, Hospital Clinic, IDIBAPS, Barcelona

${ }^{\#}$ CIBER Enfermedades Respiratorias (CIBERES), FISIB Palma de Mallorca, ${ }^{+}$Hospital Universitario Miguel Servet, Instituto Aragones Ciencias Salud, CIBER Enfermedades Respiratorias (CIBERES),

${ }^{f}$ CIBER Enfermedades Respiratorias (CIBERES), Zaragoza,

${ }^{\S}$ Hospital Arnau de Vilanova, Institut de Recerca Biomedica, Lleida, Spain. 'Dept of Sleep Medicine, The Sahlgrenska Academy, University of Gothenburg, Gothenburg, Sweden. **Dept of Internal Medicine, Unit of Respiratory Clinical Pharmacology, University of Rome "Tor Vergata", Rome, Italy.

\#\#Pulmonary and Critical Care Medical Center, Omaha, NE, USA.

CORRESPONDENCE

A. Agusti

Thorax Institute

Hospital Clinic. Villarroel 170

08036 Barcelona

Spain

E-mail: alvar.agusti@clinic.ub.es

Received:

May 192011

Accepted after revision:

June 282011

PROVENANCE

Publication of this peer-reviewed article was supported by Almirall SA Spain (article sponsor, European Respiratory Review issue 121). 
the main issues identified and discussed in the meeting moving from well-established evidence to knowledge gaps that require further research in a dimension of COPD that has been, so far, little pursued.

\section{SLEEP AND CIRCADIAN RHYTHMS IN HUMAN BIOLOGY Relevance of sleep quantity/quality for human health}

Sleep is essential in humans to maintain cognitive performance and work productivity, as well as physical, psychological and emotional wellbeing [5,6]. The acute effects of sleep deprivation include impaired cognitive, psychomotor and executive functioning. Longer term sleep deprivation appears to have additional detrimental impacts on metabolism and may contribute to the development of obesity [7]. Sleep deprivation has also been associated with the emergence of cardiovascular disease and increased all-cause mortality [5, 6, 8-10]. Sleep quality is also relevant in terms of long-term health consequences [5].

Normal human sleep follows a distinct sequence (sleep architecture) of increasingly deep sleep interrupted by periods of relative brain activity (rapid eye movement or REM sleep). Disruptions of this normal sequence of events are known as "sleep fragmentation". Restricted sleep duration and sleep fragmentation can occur due to a variety of factors, including shift work, emotional stress, sleep disorders and acute and chronic medical conditions [11-13].

The impact of sleep deprivation is well demonstrated in shift workers and in sleep restriction studies. For instance, a recent prospective 6-yr follow-up study in the Canadian province of Quebec found that adults who reported sleeping 5-6 h per night were $35 \%$ more likely to experience significant weight gain $(>5 \mathrm{~kg}$ ) during the 6-yr follow-up period than were those who reported sleeping for $7-8 \mathrm{~h}$ per night (considered a normal sleep duration for a healthy adult) [9]. In the large Nurses Health Study there was an increased risk for developing diabetes, with all the attendant health risks, and experiencing adverse coronary events in females if they reported sleeping for $\leqslant 7 \mathrm{~h}$ per night compared with those achieving an average of $8 \mathrm{~h}$ per night [10].

\section{Circadian variation of lung function in healthy subjects}

Lung function displays clear circadian variation in healthy individuals. Ventilation changes with sleep onset and with sleep stage [14-16], with a considerable reduction as individuals move from light to deep sleep [17]. These ventilatory changes result in a loss of air in the dependent (dorsal) region of the lung and are likely due to reduced respiratory muscle tone and consequent airway closure [17].

SPENGLER and SHEA [18] examined pulmonary function over $41 \mathrm{~h}$ in 10 healthy adults who remained in a state of relaxed wakefulness. They identified a diurnal rhythm in lung function with significant circadian variations in forced expiratory volume in $1 \mathrm{~s}$ (FEV1), FEV1/forced vital capacity, cortisol and core body temperature. Cholinergic tone also has a normal circadian rhythm with higher levels during the sleeping hours, and this can lead to airflow limitation in patients with COPD [19]. Conversely, changes in pulmonary function at night may also reflect changes in both cortisol levels and body temperature [18]. MORTOLA [15] found that the ventilatory response to carbon dioxide falls during sleep. This may have a significant influence on the ventilatory response during sleep [20, 21]. All in all, these changes mean that the respiratory system is less capable of responding to challenges during the night.

\section{SLEEP DISTURBANCE IN COPD Prevalence}

Epidemiological reporting of sleep disturbance and night-time symptoms in COPD is limited. However, data from a small number of studies in patients with COPD over the past $30 \mathrm{yrs}$ does indicate that these patients experience a range of sleep disturbances. In a recent study, $76 \%$ of patients reported frequent nocturnal awakening (table 1) [22-27]. Additional information from baseline evaluations of patients with COPD recruited from randomised controlled trials of respiratory drugs also highlight the prevalence of nocturnal symptoms and sleep disturbance in COPD. For example, ANZUETO et al. [30] found that, among 797 patients with COPD, 34\% reported night-time wakening due to COPD in the 7 days prior to randomisation. TASHKIN et al. [31] evaluated the efficacy of budesonide/formoterol in patients with COPD, and reported that $>50 \%$ of the 1,704 patients recruited into the study reported some degree of sleep disturbance at baseline. More recently, a pan-European survey was undertaken to specifically explore the prevalence and impact of night-time symptoms in COPD patients [32]. Night-time symptoms were assessed using the Jenkins Sleep Scale, which asks how often the patient experiences trouble falling asleep, trouble staying asleep, waking up several times in the night or waking up feeling tired and worn out after usual amount of sleep. The recall period was the previous month and patients were scored according to the number of days with sleep disturbances reported in that period. The authors found that $78.1 \%$ of patients reported some degree of night-time symptoms in relation to COPD, a much higher incidence than previous estimates of around 50\% [31]. They also reported that, as the severity of airflow limitation increased, so did the prevalence of night-time symptoms (fig. 1). Interestingly, patients reporting bothersome symptoms at night experienced more daytime breathlessness (Medical Research Council (MRC) dyspnoea scale), had more exacerbations within the previous 12 months and were receiving more maintenance medications than those who did not report bothersome night-time symptoms. The study was not designed to establish a causal relationship between these observations but it is interesting to speculate that night-time symptoms, either respiratory or sleep related, may be a marker for the emergence of uncontrolled disease, with such patients being at greater risk for an exacerbation. Further studies are needed to investigate this hypothesis prospectively. Finally, this survey showed that clinicians underestimate the prevalence of night-time symptoms related to COPD because, while $78.1 \%$ of patients reported some degree of bothersome night-time symptoms, only $67 \%$ of clinicians reported that their patients were bothered at night by their COPD [32].

\section{Pathogenesis}

Patients with COPD and night-time symptoms experience a range of changes in sleep architecture including poor sleep efficiency, increased sleep latency, decreased total sleep time, increased waking after sleep onset and decreased stage N3 and 


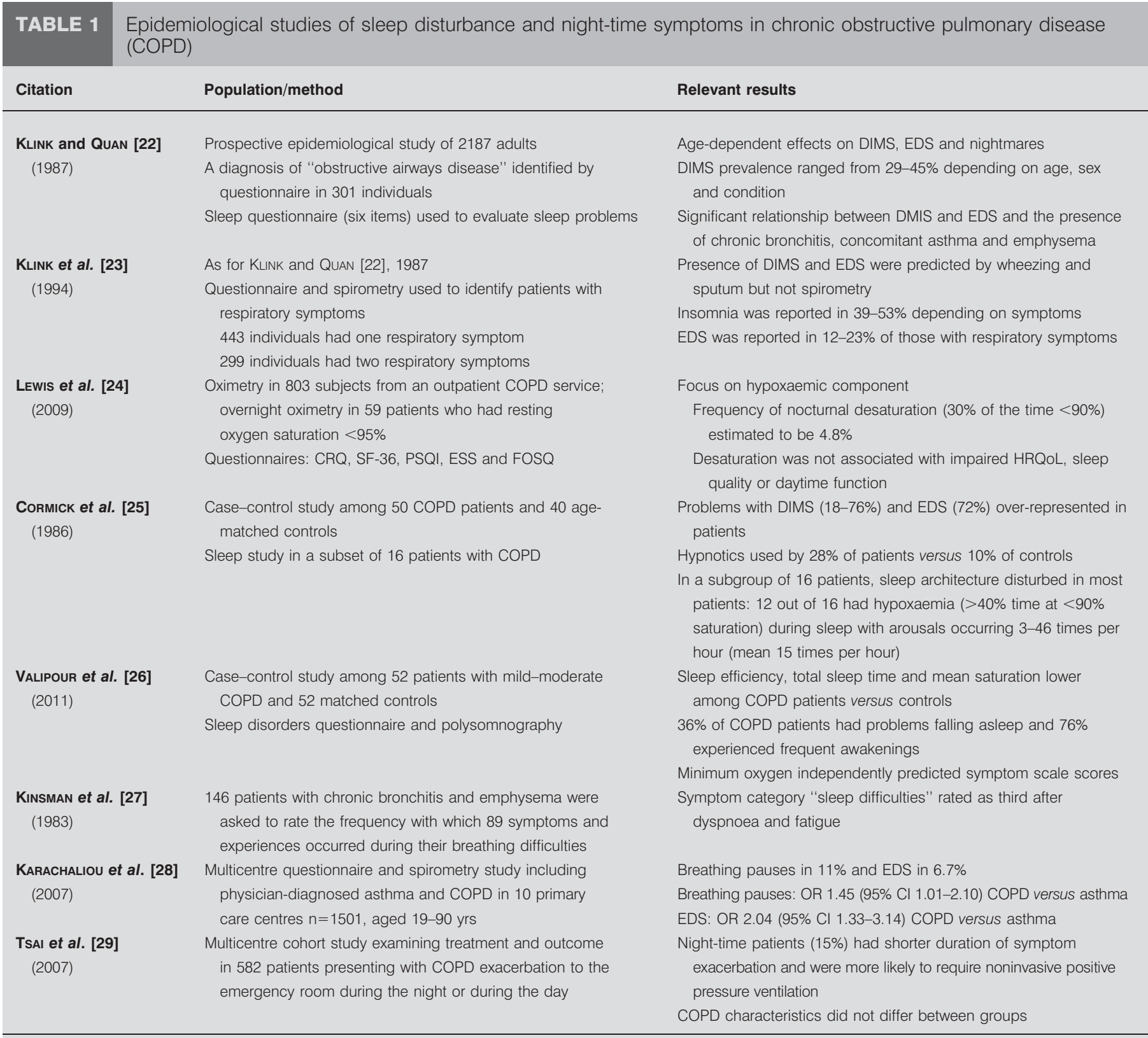

DIMS: disorders of initiating and maintaining sleep; EDS: excessive daytime sleepiness; CRQ: Chronic Respiratory Questionnaire; SF-36: Short Form-36 questionnaire PSQI: Pittsburgh Sleep Quality Index; ESS: Epworth Sleepiness Scale; FOSQ: Functional Outcomes of Sleep Questionnaire; HRQoL: health-related quality of life.

stage $\mathrm{R}$ sleep [3]. These sleep disturbances may be related to multiple causes including demographic factors, such as age (most patients with COPD are over 50 yrs of age) and presence of obesity, pharmacotherapy, disease-specific symptoms, including wheezing and cough [33], or the presence of comorbid sleep disorders (e.g. obstructive sleep apnoea (OSA)) or other medical conditions. Psychological distress related to COPD may also contribute to disturbed sleep in these patients. Moreover, altered lung function in COPD may make patients more prone to hypoxaemia, a condition that has also been shown to disrupt normal sleep architecture [34]. In fact, patients with COPD frequently experience nocturnal oxygen desaturation episodes [35]. This largely reflects the physiological impact of sleep on ventilation discussed previously, and may be aggravated by changes in tidal volume and rapid shallow breathing, since accessory breathing muscles become hypotonic during sleep [17, 36-38], particularly during periods of REM sleep [34]. All these changes, in combination with the blunted ventilatory responses to hypercapnia during sleep alluded to before [39], lead to changes in arterial blood gases during sleep in COPD [40], as recently modelled by MARRONE et al. [34]. The consequences of nocturnal hypoxaemia (and hypercapnia) in patients with COPD may include acute events, such as altered sleep structure, arrhythmias and increased pulmonary artery pressure, and long-term sequelae, which include an increased risk for cardiovascular and cerebrovascular disease (table 2) [34, 41, 42]. 


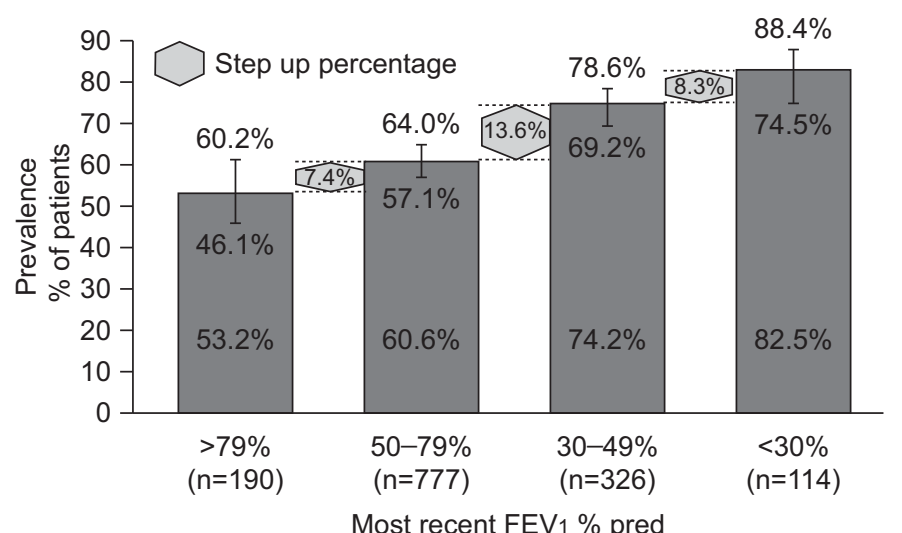

FIGURE 1. Prevalence (per cent and $95 \%$ confidence interval) of night-time symptoms among patients with chronic obstructive pulmonary disease aged $>40 \mathrm{yrs}$ with a history of smoking, stratified by forced expiratory volume in $1 \mathrm{~s}$ (FEV1) \% predicted. Data are taken from [32]

Moreover, ventricular ectopic episodes are more frequent during periods of hypoxaemia and are reduced with nocturnal oxygen therapy [43]. Yet, the evidence relating sleep hypoxaemia to arrhythmias and vascular risk is better established in patients with OSA than in patients with COPD [45].

Finally, there is an apparent bias towards nocturnal death among patients experiencing a COPD exacerbation [44]. Interestingly, this has also been reported in patients with OSA [46]. Teasing out the relative contribution of each of these potential mechanisms of sleep disturbance among patients with COPD is the challenge we now face as a prelude to determining the most appropriate management strategy.

\section{Measuring sleep disturbances in COPD}

Sleep disturbance can include a range of phenomenological entities that range from sleep disorders (such as insomnia) and disrupted sleep (exemplified by arousals/awakenings, abnormal sleep onset or duration or altered sleep architecture), to disturbed or delayed sleep due to physical symptoms such as pain, cough or sputum production. Each of them may need a specific method for assessment. Furthermore, it is important to highlight that, currently, we lack an agreed clinical and operational definition of night-time symptoms in COPD. This is a pre-requisite for the exploration and definition of the relationship between disturbed sleep and night-time symptoms in COPD. Currently, definitions of night-time symptoms in COPD include worsening of respiratory symptoms that either occur at night or interfere with sleep onset or duration, an increased need for reliever medication during the night, and sleep disturbances such as frequent nocturnal awakenings and poor quality sleep (fragmentation, arousals). All these endpoints have been used in various studies examining the epidemiology of sleep disturbances in COPD and also in studies evaluating novel pharmacological therapies, usually as a secondary end-point, and are discussed further below.

The occurrence of respiratory symptoms at night in COPD patients is usually captured using a patient-completed diary. This approach provides a useful insight into the patient's perception of the nocturnal burden of their disease, but the precision and reliability of this method of data collection is poorly defined, and no validated questionnaires are currently available. Sleep diaries generally consist of questions asking patients to record their sleep duration and recalled sleep disruptions (awakenings due to COPD symptoms). Patients may also be asked to record a sleep score that reflects the degree of perceived sleep disruption.

\section{TABLE 2 Consequences of nocturnal hypoxaemia and hypercapnia in chronic obstructive pulmonary disease (COPD)}

Parameter/outcome

Sleep structure

Arrhythmias

Pulmonary artery pressure

Diurnal blood gases

Mortality

Cardiovascular and cerebrovascular risk

Inflammatory mechanisms

Acute consequences

Chronic consequences

Altered in severe COPD, but inconsistent improvement of sleep with oxygen administration

Observed during severe sleep desaturations; prevented by oxygen administration

Increased

Inconsistent finding with regard to differences between desaturators and nondesaturators

Similar wake blood gas evolution among desaturators and nondesaturators; possible worsening of blood

gases in severe COPD suggested by beneficial effects of ventilatory treatment

Prognosis independent of nocturnal hypoxaemia measured in stable conditions

night among patients with exacerbations (not systematically evaluated)

Increased ventricular ectopic events during periods of hypoxaemia
Increased, possibly as a result of increased sympathetic activity and altered peripheral vascular tone due to recurrent nocturnal hypoxia

Data are taken from $[34,41-44]$. 
At present there are no validated biomarkers that can inform accurately on whether an individual has fulfilled their sleep need or is in sleep deficit, so that there is no way to evaluate the clinical relevance of the reported sleep durations beyond the degree of deviation from an accepted population norm for healthy adults of 7-8 h per night.

New technology may have the capacity to monitor cough during sleep in patients with COPD. Yet, there are numerous challenges in recording, analysing and quantifying cough sounds and current systems have only been tested over short time periods and in limited patient groups [47].

Sleep laboratory measurements offer a well-validated, reliable and reproducible method of collecting objective data on sleep duration and quality. These might include assessment of sleep latency (time to falling sleep) and evaluation of sleep itself using electroencephalography, electro-oculography, electromyography, respiratory flow, heart rate, oxygen and carbon dioxide levels, and leg movements. However, they are intrusive and in most cases require that patients attend a clinic for overnight recording. This makes them unsuitable for largescale randomised controlled trials of potential pharmacological interventions, but they can be useful for the evaluation of individual patients for whom conditions such as comorbid sleep apnoea syndrome are suspected.

Measuring the impact of night-time symptoms may provide the greatest scope for the objective evaluation of night-time symptoms and sleep disturbance in COPD. This may be achieved using validated scales to determine sleep duration and quality, the emergence of daytime symptoms or the impact upon health status or quality of life (QoL). The impact on sleep duration and quality can be recorded using validated tools such as the Pittsburgh Sleep Quality Index (PSQI) [48]. The PSQI has been validated in a variety of disease settings from primary insomnia to cancer [48, 49]. However, while useful, this scale is not disease-specific and does not provide information on the underlying causes of disturbed sleep and night-time awakenings. Another potential approach is to measure the emergence of daytime symptoms, such as daytime sleepiness or fatigue, related to sleep deprivation. Several validated tools are available. Daytime sleepiness can be assessed using the Epworth Sleepiness Scale [50] while fatigue can be measured using the Functional Outcomes of Sleep Questionnaire [51], the Multidimensional Fatigue Inventory [52], the Manchester COPD Fatigue Scale [53] or the Fatigue Impact Scale (COPD) [54], the latter two being specific for COPD. However, not all patients with sleep disorders express daytime symptoms and, while two clear phenotypes have been identified among patients with sleep apnoea syndrome (those with and those without daytime somnolence), no such phenotypes have been established among COPD patients.

QoL is widely used to determine the burden of disease and a wide variety of validated tools are available. The St George's Respiratory Questionnaire (SGRQ) [55] is one such tool widely used in the assessment of health-related quality of life (HRQoL) in respiratory disease and includes one question about sleep disturbances caused by cough or breathlessness. Other nondisease-specific tools that are widely used and validated measures of HRQoL include the Euro-QoL-5D (EQ-5D) [56] and Short-Form 36 (SF-36) questionnaires. The results of studies using these tools have given some indication that poor quality sleep adversely impacts HRQoL [57-59]. In a study conducted in 2009 among 30 patients with clinically stable COPD, poor sleep quality (assessed using the PSQI) was reported by $70 \%$ of patients, and HRQoL (assessed using the SGRQ) correlated with the quality of sleep, FEV1 and severity of dyspnoea [57]. In a clinical cohort study of 180 patients with COPD (78\% of whom were male), poor quality sleep (assessed using the PSQI) was an independent predictor of the SGRQ score [58]. Finally, among a cohort of 58 patients with COPD and comorbid anxiety and depression, poor HRQoL was associated with poor sleep and psychiatric morbidity [59]. Although sleep disturbances may have a considerable negative impact on QoL in patients with COPD, it is important to keep in mind that relevant confounding factors have not been well established and are likely to be multiple.

Finally, two emerging measures that may prove useful in assessing the impact of night-time symptoms and sleep disturbance in COPD are the COPD and Asthma Sleep Impact Scale (CASIS) [60] and the COPD Assessment Test (CAT) [61]. The CASIS has been validated both in asthma $(n=84)$ and COPD $(n=79)$ and has been shown to have good internal consistency, test-retest reliability and construct validity. The CAT measures the perception of the burden of disease and includes one question on sleep. CAT is extremely well correlated with the SGRQ [61].

\section{Clinical impact}

Night-time symptoms and the consequent sleep disturbance have the potential to impact a range of long-term outcomes in patients with COPD that includes lung function changes, exacerbation frequency, the emergence of cardiovascular disease, cognitive effects, depression, quality of life and mortality. Hence, there is a pressing need for further research to establish the long-term health consequences of disturbed sleep in patients with COPD as a prelude to identifying appropriate measurement tools for use in interventional studies.

With these caveats in mind, the results published by PARTRIDGE et al. [62] are of note. These investigators conducted an internet survey of 803 patients with COPD in Europe and the USA. Patients were asked at what time of day their COPD symptoms were worse than usual. $37 \%$ of patients reported that their symptoms were most troublesome in the early morning; this rose to $46 \%$ among the 289 patients with severe COPD (defined as regular use of COPD medication, a score of 3 or above on the modified MRC dyspnoea scale, and one or more COPD exacerbations in the preceding 12 months) (fig. 2). The second most bothersome moment with respect to symptoms was during the night as reported by $25 \%$ of all patients and $34 \%$ of those with severe COPD. Whether or not there a link between early morning symptoms and night-time symptoms/sleep disturbances in COPD is unclear but deserves research.

Most recently, a pan-European study recruiting $>2,400$ patients with severe COPD has shown that phlegm and cough are the most troublesome symptoms on waking, with cough and wheezing being the most troublesome symptoms at night (fig. 2) [63]. The authors also found that $20-30 \%$ of patients perceived some degree of variation in the severity of 

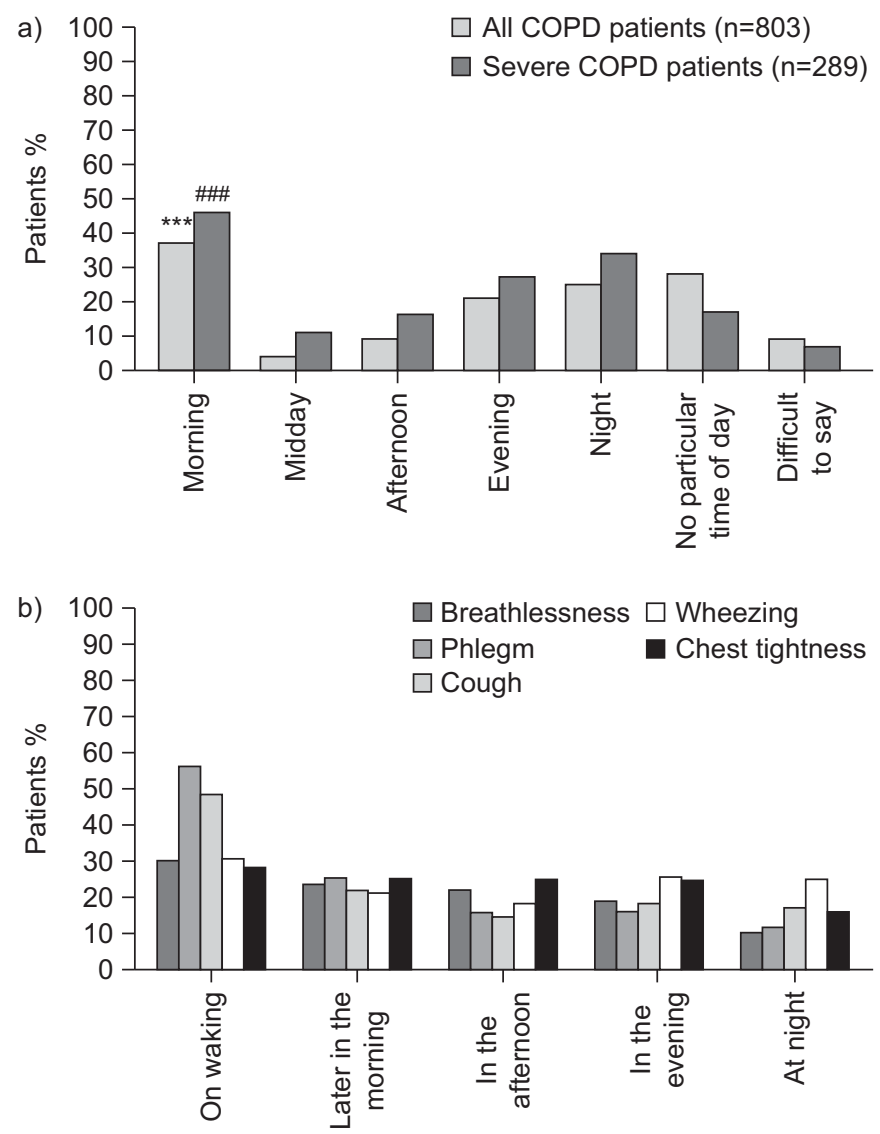

FIGURE 2. Circadian variation in the troublesomeness of a) any chronic obstructive pulmonary disease (COPD) symptom [62] and b) specific COPD symptoms. Data are taken from [63]. ${ }^{* *}: p<0.001$ versus all other times of day; \#\#\#: $p<0.001$ versus midday.

individual symptoms throughout the day, suggesting unstable airway function and raising the possibility that these patients might be at greater risk for COPD exacerbation. In fact, this study also identified a number of factors associated with reduced daily symptom variability (table 3 ), including maintenance treatment with a single long-acting bronchodilator (no versus yes; OR 2.062, 95\% CI 1.358-3.132; p=0.0007), treatment by a specialist versus a general practitioner (OR 1.390, 95\% CI $1.082-1.785 ; p=0.0099)$, and the severity of the breathlessness experienced by patients. Further research is needed to explore the relationship of night-time symptoms, daytime symptoms and risk of exacerbations.

\section{Therapeutic options}

Despite the apparent high prevalence of night-time symptoms and disturbed sleep in patients with COPD, there are only a few interventional studies that have directly targeted them.

Several studies have investigated the effects of bronchodilator therapy [19, 64-67]. In a 4-week, double-blind study among 36 patients with moderate to severe COPD, treatment with ipratropium (given four times daily) led to an improvement in oxygen saturation and also in perceived sleep quality [66]. In contrast, in another 4-week, double-blind study among 80 patients with severe stable COPD, evening dosing with tiotropium reduced the daily variation in lung function and

\begin{tabular}{|c|c|c|c|}
\hline TABLE 3 & \multicolumn{3}{|c|}{$\begin{array}{l}\text { Factors affecting daily breathlessness variation } \\
\text { in } 2,441 \text { patients with severe chronic obstructive } \\
\text { pulmonary disease }\end{array}$} \\
\hline \multicolumn{2}{|l|}{ Factor } & OR $(95 \% \mathrm{Cl})$ & p-value \\
\hline \multicolumn{2}{|c|}{ Age calculated for $10-y r$ changes } & $0.85(0.75-0.96)$ & 0.0106 \\
\hline \multicolumn{4}{|c|}{ Severity of breathlessness } \\
\hline \multicolumn{2}{|c|}{ Moderately versus a little } & $1.47(1.04-1.95)$ & 0.0083 \\
\hline \multicolumn{2}{|c|}{ Very versus a little } & $2.85(2.06-3.94)$ & $<0.0001$ \\
\hline \multicolumn{2}{|c|}{ Extremely versus a little } & $2.56(1.52-4.33)$ & 0.0004 \\
\hline \multicolumn{2}{|c|}{$\begin{array}{l}\text { Maintenance treatment with only one long- } \\
\text { acting bronchodilator (no versus yes) }\end{array}$} & $2.06(1.36-3.13)$ & 0.0007 \\
\hline \multicolumn{2}{|c|}{$\begin{array}{l}\text { Physician activity (general practitioner } \\
\text { versus specialist) }\end{array}$} & $1.39(1.08-1.78)$ & 0.0099 \\
\hline
\end{tabular}

improved nocturnal oxygen saturation but did not offer any improvement in perceived sleep quality [64]. Finally, CALVERLEY et al. [19] conducted a 6-week, double-blind, placebo-controlled study in 121 patients with stable COPD and found that the administration of tiotropium once daily in the evening did not produce more bronchodilation during the night than when it is administered once daily in the morning. Conversely, RYAN et al. [67] showed that treatment with salmeterol, a long-acting $\beta_{2^{-}}$ adrenergic bronchodilator, improves arterial oxygen saturation during sleep without significant change in sleep quality. In a randomised, double-blind, placebo-controlled, crossover study, these authors compared the effects of 4 weeks of treatment with salmeterol (50 mg b.i.d.) and matching placebo on sleeping arterial oxygen saturation $\left(\mathrm{Sa}_{2} \mathrm{O}_{2}\right)$ and sleep quality in 15 patients with moderate/severe stable COPD. They found that, both mean $\mathrm{Sa}, \mathrm{O}_{2}$ and the percentage of sleep spent below $90 \%$ of $\mathrm{Sa}, \mathrm{O}_{2}$ improved significantly with salmeterol, whereas sleep quality was similar irrespective of salmeterol or placebo.

Alternatively, various measures of sleep disturbance and nighttime symptoms have been evaluated as secondary or exploratory end-points in several randomised clinical trials of a number of respiratory drugs evaluated for the treatment of COPD (table 4) [30, 31, 68-76]. However, the evaluation of night-time symptoms was not the primary outcome in any of these trials and, besides, the tools used to assess them had not been previously validated. A recent study with aclidinium bromide, a novel long-acting anti-muscarinic drug, investigated potential effects on night-time symptoms in COPD [77]. In this 12week, randomised, double-blind, placebo-controlled study, 561 patients with COPD were treated with either aclidinium $200 \mu \mathrm{g}$ or $400 \mu \mathrm{g}$, twice daily. Overall, the results on sleep diary parameters were not statistically significantly different between the aclidinium bromide arms and placebo. However, significant differences were noted for the frequency of night-time awakenings and the ability to fall back asleep after awakening in the aclidinium bromide $400 \mu$ g group at weeks $1,4,8$ and 12 , as well as in the quality of sleep and rating of sleep in both active treatment groups at weeks 1 and 4 as compared to normal. In addition, patients reported less early morning breathlessness with active treatment (fig. 3) [77]. This study, therefore, shows 


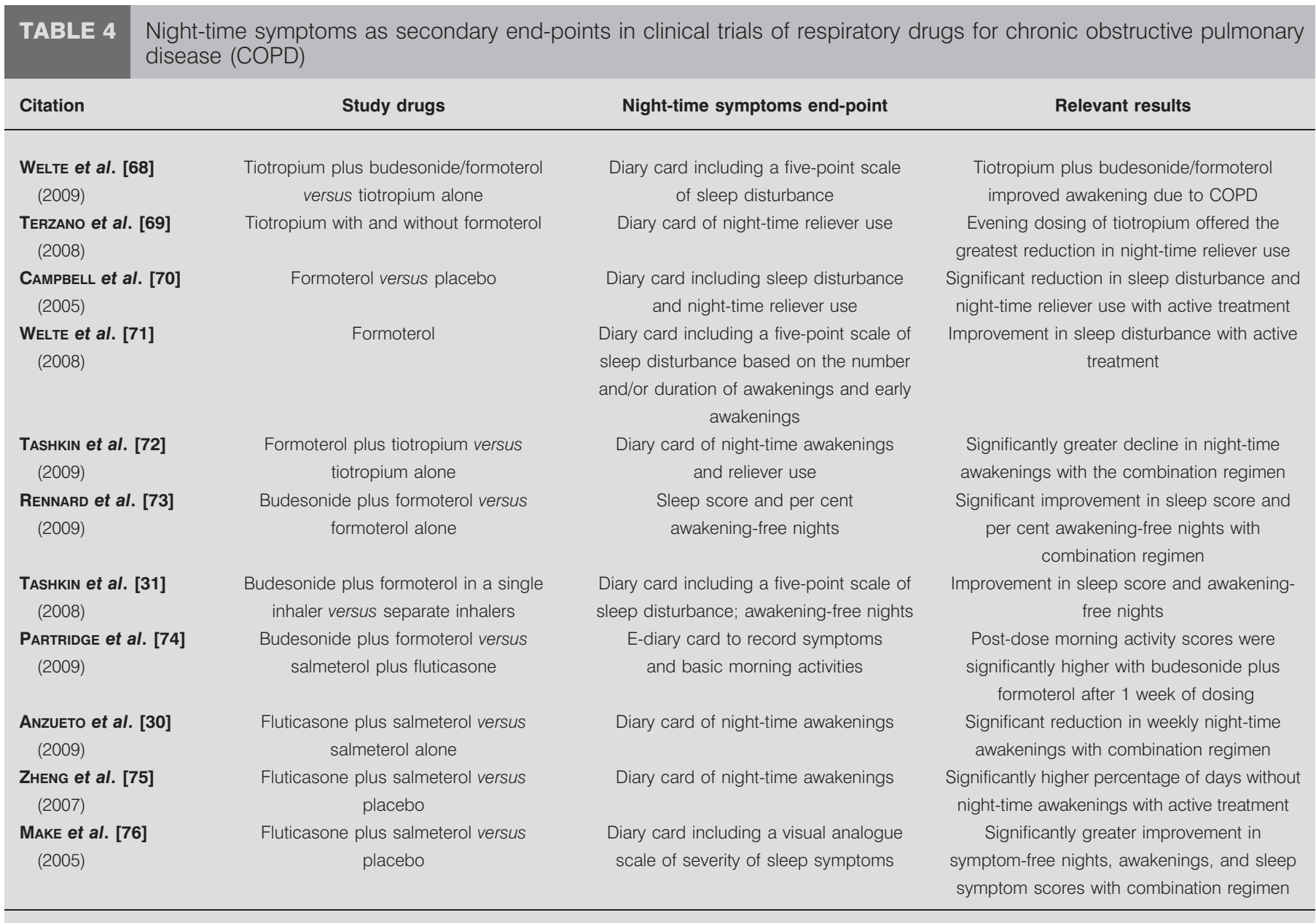

that the improvement in lung function obtained by the administration of aclidinium twice daily translates into symptomatic benefits to COPD patients, especially during sleep time and in the early morning [77].

Finally, it is well established that overnight oxygen therapy improves nocturnal oxygen saturation and pulmonary artery pressure during sleep $[78,79]$. Studies comparing noninvasive ventilation with supplemental oxygen suggest that the former can also improve quality of life, although this effect was not fully unequivocal $[80,81]$. In these studies, some proportion of patients may have suffered from concurrent sleep apnoea, relief of which may explain the observed improvements. In the study reported by McEvor et al. [81] in 2009, 144 patients with COPD were randomly allocated to either nocturnal noninvasive ventilation plus long-term oxygen therapy $(n=72)$ or to longterm oxygen therapy alone $(n=72)$. No differences in lung function or overall survival were observed, but patients treated with noninvasive ventilation reported significantly better general health (SF-36 median score of 32 points with long-term oxygen therapy versus a median score of 20 with noninvasive ventilation; $\mathrm{p}=0.0002$ ) and mental health (median scores of 80 and 70 , respectively; $p=0.009$ ) [81]. However, using the Profile of Mood States assessment, patients receiving noninvasive ventilation reported significantly more confusion and bewilderment $(p=0.020)$ and significantly less vigour $(p=0.050)$ [81].

\section{LESSONS FROM OTHER MEDICAL CONDITIONS}

Sleep disturbance is common in a range of chronic diseases including restrictive lung diseases and asthma, sleep apnoea syndrome, cardiovascular disease including heart failure [82],

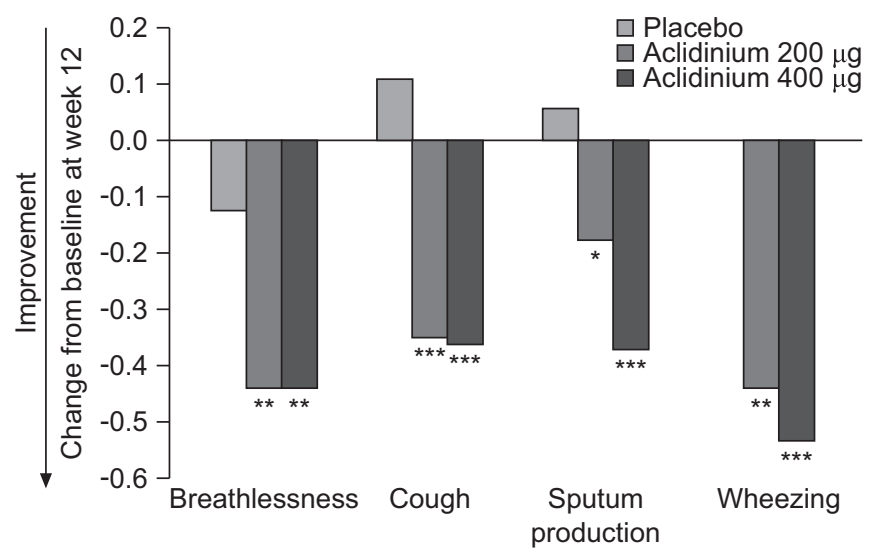

FIGURE 3. Severity and impact of night-time symptoms among patients with chronic obstructive pulmonary disease treated with aclidinium bromide or placebo. The frequency of each variable was scored as follows: 0 , never; 1, 1-2 times; 2, 3-4 times; $3,5-6$ times; and $4, \geqslant 7$ times. Weekly averages were analysed. *: $p<0.05$; $* *: p<0.01 ; * * *: p<0.001$ versus placebo. Reproduced from [77] with permission from the publisher. 
gastrointestinal disorders, pain disorders, renal disorders and cancer [3, 83-85] over and above levels observed in agematched general populations. Research is now ongoing in a number of fields to better understand the reciprocal relationship between chronic disease and sleep disturbances, as well as the implications for both day to day management and longterm outcomes. Some lessons from these fields may be of interest for a better understanding of night-time symptoms in COPD patients.

\section{The overlap syndrome: COPD and sleep apnoea}

COPD and sleep disordered breathing (mainly OSA) are two of the most common respiratory disorders and frequently coincide [35]. Although good epidemiological data is currently lacking, the prevalence of sleep apnoea among patients with COPD is likely to be similar to that seen in the general population (about $4 \%$ ). Sleep studies to diagnose OSA in patients with COPD are not routinely indicated unless the patient presents with additional symptoms, such as snoring and/or excessive daytime somnolence, that suggest the presence of OSA [4].

The co-occurrence of OSA and COPD is referred to as overlap syndrome. Although it probably affects a relatively small proportion of patients, the impact on sleep and long-term health can be profound. Patients with the overlap syndrome experience more severe nocturnal oxygen desaturation than patients with either condition alone, and are at considerably greater risk of death [35]. As in patients with OSA, sleep fragmentation in patients with overlap syndrome depends mainly on the sleep apnoea component, and it is thought to be responsible for their excessive daytime sleepiness. Whether the density of arousals during sleep due to respiratory events is also related with major outcomes in patients with overlap syndrome is not yet known. The precise mechanisms that underlie this increased mortality rate are not fully defined but they are most likely related to the adverse haemodynamic effects and prolonged activation of inflammatory responses by hypoxia [86] leading to an increased risk for cardiovascular events [35]. Interestingly, patients with overlap syndrome appear to experience more frequent exacerbations and a more rapid decline in lung function [87, 88] but, again, further research is required to define and understand this relationship more fully.

Appropriate treatment strategies for patients with overlap syndrome are emerging. A recent study has shown that the use of continuous positive airway pressure (CPAP) to treat OSA in patients with COPD dramatically improves outcomes in these latter patients, including COPD exacerbation frequency and overall survival [89]. In this study, among a population of 4,241 snorers referred to a sleep clinic between 1996 and 2000, 3,102 patients were diagnosed with OSA, of whom 598 were also diagnosed with COPD. Of these latter 598 patients, 228 with overlap syndrome were treated with CPAP whereas 213 did not receive CPAP. A further 210 patients with COPD without OSA were followed as a comparator group. Figure 4 illustrates the improvement in both survival $(p<0.001)$ and exacerbation frequency $(p<0.001)$ for patients with overlap syndrome treated with CPAP. Whether patients with COPD alone might have benefited further from CPAP therapy was not evaluated. a) Overall survival

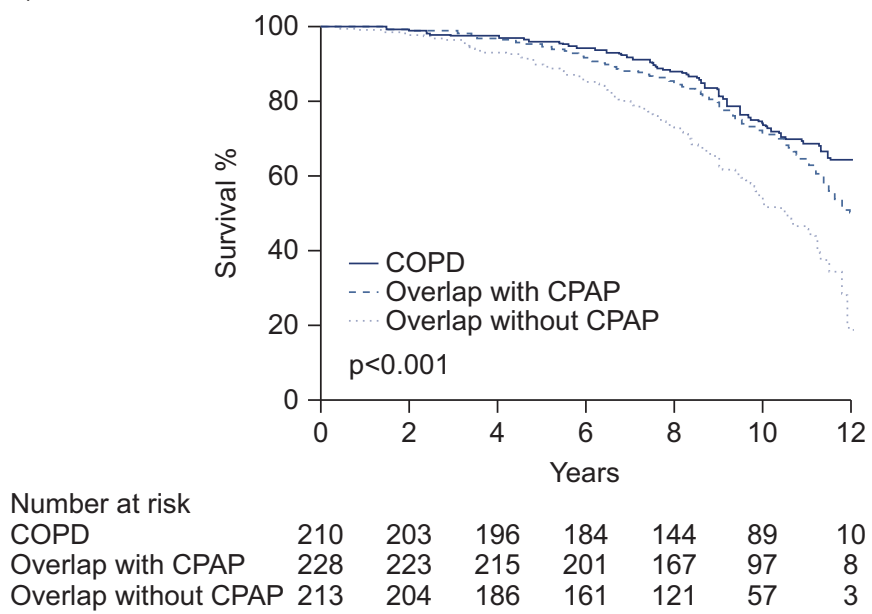

b) Exacerbation frequency

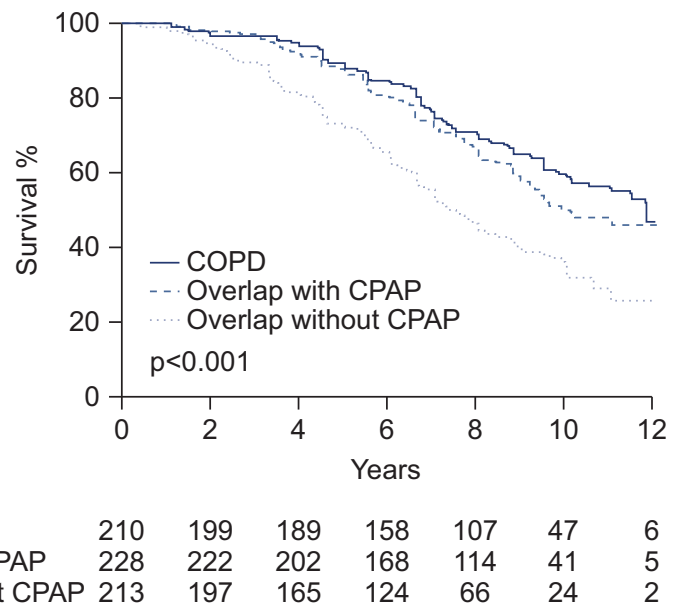

FIGURE 4. Outcomes (survival and exacerbation frequency) among patients with overlap syndrome treated with continuous positive airway pressure (CPAP). COPD: chronic obstructive pulmonary disease. Reproduced from [89] with permission from the publisher.

\section{Asthma}

Nocturnal symptoms of asthma negatively impact sleep quality as disease control declines [90]. This was clearly illustrated in a survey of 1,199 patients with asthma (mean age $46.5 \mathrm{yrs}$ ), $75.3 \%$ of whom had well controlled asthma (according to the Asthma Therapy Assessment Questionnaire (ATAQ) score 0; $\mathrm{n}=146$ ) and reported no difficulty in getting a good night's sleep. This proportion was lower (55.7\%) among those with less well controlled asthma (ATAQ score 1-2; $n=923$ ), particularly among those $(6.9 \%)$ with very poorly controlled asthma (ATAQ score 3-4; $\mathrm{n}=130$ ).

Insomnia and sleep-related symptoms, such as excessive daytime sleepiness, are more common in asthmatic females than males [91]. Among 470 subjects with current asthma from the Nordic European countries who took part in the European Community Respiratory Health Survey II, females were significantly more likely than males to report insomnia $(p<0.0001)$ and excessive daytime sleepiness $(p<0.0001)$, and female sex was a significant independent determinant for 
insomnia (adjusted OR 3.67, 95\% CI 1.94-6.95) and excessive daytime sleepiness (adjusted OR 2.53, 95\% CI 1.32-4.86). One possible explanation may be that females present later for treatment than males, such that their symptoms are worse by the time they reach their clinician. There are currently no data to determine whether such a sex difference is also apparent in COPD patients, but this is an area that warrants further research given that females who smoke may be more susceptible to the development of the disease than males [92].

As observed in COPD, individual symptoms appear to vary during the day among patients with asthma. For example, cough appears to peak shortly after waking [93]. Why this should be is unclear but it appears that sleep suppresses cough [93]. Whether this suppression of the cough reflex is also apparent in COPD patients is also unclear given that cough is reported by patients as one of the most troublesome nocturnal symptoms [63].

Several studies have documented that inflammation worsens in individuals who have nocturnal asthma during the nighttime hours, as compared with those who have no nocturnal asthma of comparable asthma severity [94-97]. These inflammatory changes have been related to airflow limitation and reversibility [98]. Most investigators view the circadian cycles of cortisol, cholinergic tone, histamine and adrenaline as having theoretical relevance to inflammation, but the specific and detailed mechanistic links among these cycling biological processes and the control of inflammation remain incompletely defined [99].

\section{CONCLUSIONS AND FUTURE DIRECTIONS}

Following the discussion of the available evidence presented above, the panel concluded that:

1) While the term "night-time symptoms" in COPD has not been distinctly defined in an objective sense, available epidemiological data suggest that nocturnal symptoms and symptomatic sleep disturbance is common, and may exceed $75 \%$, among patients with COPD. Night-time symptoms and sleep disturbance are very likely to be clinically underestimated and, certainly, are not a focus of current management guidelines for the disease. Given our current understanding of the negative long-term health consequences of sleep disturbance in healthy adults, maintaining and promoting adequate sleep by reducing the severity of the night-time symptoms needs to be considered as a novel therapeutic clinical goal for patients with COPD.

2) Current definitions of night-time symptoms in COPD span both respiratory and other symptoms of the disease itself, as well as symptoms of disturbed sleep such as frequent nocturnal awakenings, difficulty falling back to sleep after an awakening and shortened sleep duration. In order to explore appropriate management strategies to improved disturbed sleep in patients with COPD it may be necessary to operationally separate night-time symptoms related to the disease from sleep disturbance.

3) The "troublesomeness" of the classic symptoms of COPD differ in the morning and at night; the most troublesome symptoms in the morning are phlegm and cough, while at night they are wheezing and chest tightness. However, there is insufficient epidemiological data at present to speculate on whether there are different phenotypes of patients among the population of patients with COPD with regard to night-time symptoms. Indeed there is currently a paucity of data on sleep disturbance and circadian variation in lung function, comorbid conditions and the relationships among them in patients with COPD. For the future, it will be necessary to explore the most appropriate way to deploy respiratory and other drugs in order to manage night-time symptoms and sleep disorders in patients with COPD.

4) It will also be necessary to explore the potential links between night-time symptoms and long-term clinical outcomes to ensure that any interventions aimed at acutely improved night-time symptoms and/or sleep disturbance in COPD also improve or stabilise the long-term health of patients with COPD. While subjective sleep measures are informative, objective measures providing quantifiable data (e.g. actimetry) will be needed to establish whether there is a relationship between night-time symptoms and long-term outcomes in COPD. Relevant clinical outcomes in this respect might include all-cause mortality, the frequency of adverse cardiovascular events, the presence and severity of day and night-time symptoms, exacerbation frequency and improvement in quality of life, among others.

In summary, night-time symptoms in COPD are prevalent, bothersome and likely to adversely impact long-term outcomes for patients, but they are often not reported and/or considered in the clinical management of the disease. This "forgotten" dimension warrants further clinical investigation with validated tools.

\section{STATEMENT OF INTEREST}

This study was supported by an unrestricted educational grant from Almirall S.A., Barcelona, Spain. A. Agusti has received honoraria for participating in advisory boards, lecturing at meetings and/or funds for research in the field of COPD from Almirall, AstraZeneca, Boehringer Ingelheim, Chiesi, Esteve, GSK, MSD, Novartis, Nycomed, Pfizer, Roche, and Procter and Gamble. J. Hedner has received fees for speaking at research symposia by Weinmann $\mathrm{GmbH}$ and a fee for consulting with Almirall S.A. He has received two unrestricted grants from ResMed Inc. and Philips Respironics for maintenance of the European Sleep Apnea Database (ESADA). J.M. Marin has received fees for speaking (less than $€ 1,000$ ), and has received public funds for research from the Institut Carlos III, Madrid, Spain. M. Cazzola has received financial support for research and attending meetings and fees for speaking and consulting from Abbott, Almirall, AstraZeneca, Boehringer Ingelheim, Chiesi Farmaceutici, Dey, Eli Lilly, Gentili, GSK, Lallemand Pharma, Meda, Menarini Farmaceutici, Novartis, Nycomed, Pfizer, Sanovel, Sanofi-Aventis, Sigma-Tau and Valeas. S. Rennard has served as a consultant or participated in advisory boards for: ABIM, Able Associates, Adelphi Research, Almirall, APT, Aradigm, Argenta, AstraZeneca, BI (ACCP), Biostrategies, BoomCom, Britnall and Nicolini, Capital Research, Chiesi, Clinical Advisors, CommonHealth, Complete Medical Group, Consult Complete, COPDForum, DataMonitor, Decision Resources, Defined Health, Dey, Dunn Group, Easton Associates, Enterprise Analysis, Equinox, Forest, Fulcrum, Gerson Lehman, GSK, Guidepoint, Hoffman LaRoche, IMS, Informed, Inspire, Insyght, KOL Connection, Leerink Swan, M. Pankove, MDRx Financial, MedaCorp, Medimmune, Mpex, Novartis, Nycomed, Oriel, Otsuka, Pearl, Pennside Partners, Pfizer, Pharma Ventures, Pharmaxis, Pick Research, Prescott, Price Waterhouse, Propagate, Pulmatrix, Pulmonary Reviews, Quadrant, Reckner Associates, Recruiting Resource, Reviews and Trends in COPD/Convergent Health Solutions, Roche, Sacoor, Schering, Schlesinger Medical, Scimed, Smith 
Research, Sudler and Hennessey, Talecris, Theravance, UBC and Uptake Medical, Vantage Point; has received lecture fees from AAAAI, Am Col Osteopathic Physicians, Asan Medical Center, ATS, AstraZeneca, California Soc Allergy, Convergent Health Solutions for Reviews and Trends in COPD, COPDFoundation, Creative Educational Concepts, Dey, Duke, France Foundation, Information TV, University of California-Los Angeles, Network for Continuing Education, Novartis, Nycomed, Otsuka, Pfizer, Sarasota Mem Hospital, Spanish Thoracic Society, University of Washington, University of Alabama-Birmingham, University of Pittsburgh, University of British Columbia, University of California-Davis and VA Sioux Falls; and has received industry sponsored grants from AstraZeneca, Biomarck, Centocor, GlaxoSmithKline, Mpex, Nabi, Novartis, Otsuka and Pfizer. S. Rennard has also received tobacco industry funding, specifically from the tobacco industry for studies relating to harm reduction and to the impact of tobacco smoke on stem cells, and has also consulted with RJ Reynolds, without personal fee, on the topic of harm reduction. He received funding from RJ Reynolds to evaluate the effect of a harm reduction product in normal smokers (1996) and in subjects with chronic bronchitis (1999) and to assess the effect of smoking cessation on lower respiratory tract inflammation (2000); participated in a Philip Morris multicentre study to assess biomarkers of smoke exposure (2002); received funding for a clinical trial from the Institute for Science and Health (2005), which receives support from the tobacco industry, to evaluate biomarkers in exhaled breath associated with smoking cessation and reduction (this study was supplemented with funding from Lorillard and RJ Reynolds); received a grant from the Philip Morris External Research Program (2005) to assess the impact of cigarette smoking on circulating stem cells in the mouse. He has consulted with RJ Reynolds on the topic of harm reduction until 2007, but did not receive personal remuneration for this. S. Rennard is not currently involved in tobacco-industry funded projects; all ties with tobacco industry companies and entities supported by tobacco companies were terminated in 2007.

\section{ACKNOWLEDGEMENTS}

We thank T. Lonergan from Complete Medical Communications (Macclesfield, UK) who provided medical writing support funded by Almirall S.A., Barcelona, Spain.

\section{REFERENCES}

1 Agusti A, Calverley PM, Celli B, et al. Characterisation of COPD heterogeneity in the ECLIPSE cohort. Respir Res 2010; 11: 122.

2 American Thoracic Society, European Respiratory Society. Standards for the Diagnosis and Management of Patients with COPD. New York, American Thoracic Society; Lausanne, European Respiratory Society, 2004.

3 Parish JM. Sleep-related problems in common medical conditions. Chest 2009; 135: 563-572.

4 Global Initiative for Chronic Obstructive Lung Disease. Global Strategy for Diagnosis, Management, and Prevention of COPD. Global Initiative for Chronic Obstructive Lung Disease, 2010.

5 Banks S, Dinges DF. Behavioural and physiological consequences of sleep restriction. J Clin Sleep Med 2007; 3: 519-528.

6 Balkin TJ, Rupp T, Picchioni D, et al. Sleep loss and sleepiness: current issues. Chest 2008; 134: 653-660.

7 Morselli L, Leproult R, Balbo M, et al. Role of sleep duration in the regulation of glucose metabolism and appetite. Best Pract Res Clin Endocrinol Metab 2010; 24: 687-702.

8 Knutson KL, Spiegel K, Penev P, et al. The metabolic consequences of sleep deprivation. Sleep Med Rev 2007; 11: 163-178.

9 Chaput JP, Despres JP, Bouchard C, et al. The association between sleep duration and weight gain in adults: a 6-year prospective study from the Quebec Family Study. Sleep 2008; 31: 517-523.
10 Patel SR, Ayas NT, Malhotra MR, et al. A prospective study of sleep duration and mortality risk in women. Sleep 2004; 27: 440-444.

11 Akerstedt T, Kecklund G, Gillberg M. Sleep and sleepiness in relation to stress and displaced work hours. Physiol Behav 2007; 92: 250-255.

12 Mezick EJ, Matthews KA, Hall M, et al. Intra-individual variability in sleep duration and fragmentation: associations with stress. Psychoneuroendocrinology 2009; 34: 1346-1354.

13 van den Berg JF, Knvistingh NA, Tulen JH, et al. Actigraphic sleep duration and fragmentation are related to obesity in the elderly: the Rotterdam Study. Int J Obes (Lond) 2008; 32: 1083-1090.

14 Mohsenin V. Sleep in chronic obstructive pulmonary disease. Semin Respir Crit Care Med 2005; 26: 109-116.

15 Mortola JP. Breathing around the clock: an overview of the circadian pattern of respiration. Eur J Appl Physiol 2004; 91: 119-129.

16 Collop NA, Salas RE, Delayo M, et al. Normal sleep and circadian processes. Crit Care Clin 2008; 24, 449-460: v.

17 Appelberg J, Pavlenko T, Bergman H, et al. Lung aeration during sleep. Chest 2007; 131: 122-129.

18 Spengler CM, Shea SA. Endogenous circadian rhythm of pulmonary function in healthy humans. Am J Respir Crit Care Med 2000; 162: 1038-1046.

19 Calverley PM, Lee A, Towse L, et al. Effect of tiotropium bromide on circadian variation in airflow limitation in chronic obstructive pulmonary disease. Thorax 2003; 58: 855-860.

20 Phillipson EA, Sullivan CE, Read DJ, et al. Ventilatory and waking responses to hypoxia in sleeping dogs. J Appl Physiol 1978; 44: 512-520.

21 Phillipson EA, Murphy E, Kozar LF. Regulation of respiration in sleeping dogs. J Appl Physiol 1976; 40: 688-693.

22 Klink M, Quan SF. Prevalence of reported sleep disturbances in a general adult population and their relationship to obstructive airways diseases. Chest 1987; 91: 540-546.

23 Klink ME, Dodge R, Quan SF. The relation of sleep complaints to respiratory symptoms in a general population. Chest 1994; 105: 151-154.

24 Lewis CA, Fergusson W, Eaton T, et al. Isolated nocturnal desaturation in COPD: prevalence and impact on quality of life and sleep. Thorax 2009; 64: 133-138.

25 Cormick W, Olson LG, Hensley MJ, et al. Nocturnal hypoxaemia and quality of sleep in patients with chronic obstructive lung disease. Thorax 1986; 41: 846-854.

26 Valipour A, Lavie P, Lothaller $\mathrm{H}$, et al. Sleep profile and symptoms of sleep disorders in patients with stable mild to moderate chronic obstructive pulmonary disease. Sleep Med 2011; 12: 367-372.

27 Kinsman RA, Yaroush RA, Fernandez E, et al. Symptoms and experiences in chronic bronchitis and emphysema. Chest 1983; 83: 755-761.

28 Karachaliou F, Kostikas K, Pastaka C, et al. Prevalence of sleeprelated symptoms in a primary care population - their relation to asthma and COPD. Prim Care Respir J 2007; 16: 222-228.

29 Tsai CL, Brenner BE, Camargo CA Jr. Circadian-rhythm differences among emergency department patients with chronic obstructive pulmonary disease exacerbation. Chronobiol Int 2007; 24: 699-713.

30 Anzueto A, Ferguson GT, Feldman G, et al. Effect of fluticasone propionate/salmeterol $(250 / 50)$ on COPD exacerbations and impact on patient outcomes. COPD 2009; 6: 320-329.

31 Tashkin DP, Rennard SI, Martin P, et al. Efficacy and safety of budesonide and formoterol in one pressurized metered-dose inhaler in patients with moderate to very severe chronic obstructive pulmonary disease: results of a 6-month randomized clinical trial. Drugs 2008; 68: 1975-2000.

32 Price D, Small M, Milligan G. The prevalence and impact of nighttime symptoms in COPD patients - results from a crosssectional study in five European countries. Proceedings of the IV 
World Asthma and COPD Forum (Paris, France, April 30 to May 3, 2011).

33 Weitzenblum E, Chaouat A. Sleep and chronic obstructive pulmonary disease. Sleep Med Rev 2004; 8: 281-294.

34 Marrone O, Salvaggio A, Insalaco G. Respiratory disorders during sleep in chronic obstructive pulmonary disease. Int J Chron Obstruct Pulmon Dis 2006; 1: 363-372.

35 Owens RL, Malhotra A. Sleep-disordered breathing and COPD: the overlap syndrome. Respir Care 2010; 55: 1333-1344.

36 Becker HF, Piper AJ, Flynn WE, et al. Breathing during sleep in patients with nocturnal desaturation. Am J Respir Crit Care Med 1999; 159: 112-118.

37 Hudgel DW, Martin RJ, Capehart M, et al. Contribution of hypoventilation to sleep oxygen desaturation in chronic obstructive pulmonary disease. J Appl Physiol 1983; 55: 669-677.

38 White JE, Drinnan MJ, Smithson AJ, et al. Respiratory muscle activity during rapid eye movement (REM) sleep in patients with chronic obstructive pulmonary disease. Thorax 1995; 50: 376-382.

39 Scano G, Spinelli A, Duranti R, et al. Carbon dioxide responsiveness in COPD patients with and without chronic hypercapnia. Eur Respir J 1995; 8: 78-85.

40 Koo KW, Sax DS, Snider GL. Arterial blood gases and pH during sleep in chronic obstructive pulmonary disease. Am J Med 1975; 58 : 663-670.

41 Mueller PT, Gomes MD, Viegas CA, et al. Systemic effects of nocturnal hypoxemia in patients with chronic obstructive pulmonary disease without obstructive sleep apnoea syndrome. J Bras Pneumol 2008; 34: 567-574.

42 Gilmartin GS, Tamisier R, Curley M, et al. Ventilatory, hemodynamic, sympathetic nervous system, and vascular reactivity changes after recurrent nocturnal sustained hypoxia in humans. Am J Physiol Heart Circ Physiol 2008; 295: H778-H785.

43 Tirlapur VG, Mir MA. Nocturnal hypoxemia and associated electrocardiographic changes in patients with chronic obstructive airways disease. N Engl J Med 1982; 306: 125-130.

44 McNicholas WT, Fitzgerald MX. Nocturnal deaths among patients with chronic bronchitis and emphysema. Br Med J (Clin Res Ed) 1984; 289: 878.

45 Gami AS, Somers VK. Implications of obstructive sleep apnoea for atrial fibrillation and sudden cardiac death. J Cardiovasc Electrophysiol 2008; 19: 997-1003.

46 Gami AS, Howard DE, Olson EJ, et al. Day-night pattern of sudden death in obstructive sleep apnoea. N Engl J Med 2005; 352: $1206-1214$.

47 Smith J. Monitoring chronic cough: current and future techniques. Expert Rev Respir Med 2010; 4: 673-683.

48 Backhaus J, Junghanns K, Broocks A, et al. Test-retest reliability and validity of the Pittsburgh Sleep Quality Index in primary insomnia. J Psychosom Res 2002; 53: 737-740.

49 Beck SL, Schwartz AL, Towsley G, et al. Psychometric evaluation of the Pittsburgh Sleep Quality Index in cancer patients. J Pain Symptom Manage 2004; 27: 140-148.

50 Johns MW. A new method for measuring daytime sleepiness: the Epworth sleepiness scale. Sleep 1991; 14: 540-545.

51 Weaver TE, Laizner AM, Evans LK, et al. An instrument to measure functional status outcomes for disorders of excessive sleepiness. Sleep 1997; 20: 835-843.

52 Stein KD, Jacobsen PB, Blanchard CM, et al. Further validation of the multidimensional fatigue symptom inventory-short form. J Pain Symptom Manage 2004; 27: 14-23.

53 Al-shair K, Kolsum U, Berry P, et al. Development, dimensions, reliability and validity of the novel Manchester COPD fatigue scale. Thorax 2009; 64: 950-955.

54 Theander $\mathrm{K}$, Cliffordson $\mathrm{C}$, Torstensson $\mathrm{O}$, et al. Fatigue Impact Scale: its validation in patients with chronic obstructive pulmonary disease. Psychol Health Med 2007; 12: 470-484.
55 Bouchet C, Guillemin F, Hoang Thi TH, et al. Validation du questionnaire St Georges pour mesurer la qualité de vie chez les insuffisants respiratoires chroniques [Validation of the St George's questionnaire for measuring the quality of life in patients with chronic obstructive pulmonary disease]. Rev Mal Respir 1996; 13: 43-46.

56 Rabin R, de Charro F. EQ-5D: a measure of health status from the EuroQol Group. Ann Med 2001; 33: 337-343.

57 Nunes DM, Mota RM, de Pontes Neto OL, et al. Impaired sleep reduces quality of life in chronic obstructive pulmonary disease. Lung 2009; 187: 159-163.

58 Scharf SM, Maimon N, Simon-Tuval T, et al. Sleep quality predicts quality of life in chronic obstructive pulmonary disease. Int J Chron Obstruct Pulmon Dis 2011; 6: 1-12.

59 Hynninen MJ, Pallesen S, Nordhus IH. Factors affecting health status in COPD patients with co-morbid anxiety or depression. Int J Chron Obstruct Pulmon Dis 2007; 2: 323-328.

60 Pokrzywinski RF, Meads DM, McKenna SP, et al. Development and psychometric assessment of the COPD and Asthma Sleep Impact Scale (CASIS). Health Qual Life Outcomes 2009; 7: 98.

61 Jones PW, Harding G, Berry P, et al. Development and first validation of the COPD Assessment Test. Eur Respir J 2009; 34: 648-654.

62 Partridge MR, Karlsson N, Small IR. Patient insight into the impact of chronic obstructive pulmonary disease in the morning: an internet survey. Curr Med Res Opin 2009; 25: 2043-2048.

63 Kessler R, Partridge MR, Miravitlles M, et al. Symptom variability in patients with severe COPD: a pan-European cross-sectional study. Eur Respir J 2011; 37: 264-272.

64 McNicholas WT, Calverley PM, Lee A, et al. Long-acting inhaled anticholinergic therapy improves sleeping oxygen saturation in COPD. Eur Respir J 2004; 23: 825-831.

65 Mulloy E, McNicholas WT. Theophylline improves gas exchange during rest, exercise, and sleep in severe chronic obstructive pulmonary disease. Am Rev Respir Dis 1993; 148: 1030-1036.

66 Martin RJ, Bartelson BL, Smith P, et al. Effect of ipratropium bromide treatment on oxygen saturation and sleep quality in COPD. Chest 1999; 115: 1338-1345.

67 Ryan S, Doherty LS, Rock C, et al. Effects of salmeterol on sleeping oxygen saturation in chronic obstructive pulmonary disease. Respiration 2010; 79: 475-481.

68 Welte T, Miravitlles M, Hernandez P, et al. Efficacy and tolerability of budesonide/formoterol added to tiotropium in patients with chronic obstructive pulmonary disease. Am J Respir Crit Care Med 2009; 180: 741-750.

69 Terzano C, Petroianni A, Conti V, et al. Rational timing of combination therapy with tiotropium and formoterol in moderate and severe COPD. Respir Med 2008; 102: 1701-1707.

70 Campbell M, Eliraz A, Johansson G, et al. Formoterol for maintenance and as-needed treatment of chronic obstructive pulmonary disease. Respir Med 2005; 99: 1511-1520.

71 Welte T, Metzenauer P, Hartmann U. Once versus twice daily formoterol via Novolizer for patients with moderate to severe COPD-a double-blind, randomised, controlled trial. Pulm Pharmacol Ther 2008; 21: 4-13.

72 Tashkin DP, Pearle J, Iezzoni D, et al. Formoterol and tiotropium compared with tiotropium alone for treatment of COPD. COPD 2009; 6: 17-25.

73 Rennard SI, Tashkin DP, McElhattan J, et al. Efficacy and tolerability of budesonide/formoterol in one hydrofluoroalkane pressurized metered-dose inhaler in patients with chronic obstructive pulmonary disease: results from a 1-year randomized controlled clinical trial. Drugs 2009; 69: 549-565.

74 Partridge MR, Schuermann W, Beckman O, et al. Effect on lung function and morning activities of budesonide/formoterol versus salmeterol/fluticasone in patients with COPD. Ther Adv Respir Dis 2009; 3: 1-11. 
75 Zheng JP, Yang L, Wu YM, et al. The efficacy and safety of combination salmeterol $(50 \mathrm{microg}) /$ fluticasone propionate (500 microg) inhalation twice daily via accuhaler in Chinese patients with COPD. Chest 2007; 132: 1756-1763.

76 Make B, Hanania NA, ZuWallack R, et al. The efficacy and safety of inhaled fluticasone propionate/salmeterol and ipratropium/ albuterol for the treatment of chronic obstructive pulmonary disease: an eight-week, multicenter, randomized, double-blind, double-dummy, parallel-group study. Clin Ther 2005; 27: 531-542.

77 Kerwin E, Rennard SI, Gelb AF, et al. Twice-daily aclidinium bromide in COPD patients: nighttime symptoms and rescue medication use in ACCORD COPD I. Am J Respir Crit Care Med 2011; 183: A1592.

78 Fletcher EC, Levin DC. Cardiopulmonary hemodynamics during sleep in subjects with chronic obstructive pulmonary disease. The effect of short- and long-term oxygen. Chest 1984; 85: 6-14.

79 Goldstein RS, Ramcharan V, Bowes G, et al. Effect of supplemental nocturnal oxygen on gas exchange in patients with severe obstructive lung disease. N Engl J Med 1984; 310: 425-429.

80 Clini E, Sturani C, Rossi A, et al. The Italian multicentre study on noninvasive ventilation in chronic obstructive pulmonary disease patients. Eur Respir J 2002; 20: 529-538.

81 McEvoy RD, Pierce RJ, Hillman D, et al. Nocturnal non-invasive nasal ventilation in stable hypercapnic COPD: a randomised controlled trial. Thorax 2009; 64: 561-656.

82 Pedrosa RP, Lima SG, Drager LF, et al. Sleep quality and quality of life in patients with hypertrophic cardiomyopathy. Cardiology 2010; 117: 200-206.

83 Lanier BQ, Nayak A. Prevalence and impact of nighttime symptoms in adults and children with asthma: a survey. Postgrad Med 2008; 120: 58-66.

84 Drewes AM, Svendsen L, Taagholt SJ, et al. Sleep in rheumatoid arthritis: a comparison with healthy subjects and studies of sleep/ wake interactions. Br J Rheumatol 1998; 37: 71-81.

85 Tachibana N, Howard RS, Hirsch NP, et al. Sleep problems in multiple sclerosis. Eur Neurol 1994; 34: 320-323.

86 Eltzschig HK, Carmeliet P. Hypoxia and inflammation. $N$ Engl J Med 2011; 364: 656-665.
87 Donaldson GC, Seemungal TA, Bhowmik A, et al. Relationship between exacerbation frequency and lung function decline in chronic obstructive pulmonary disease. Thorax 2002; 57: 847-852.

88 Soler-Cataluna JJ, Martinez-Garcia MA, Roman SP, et al. Severe acute exacerbations and mortality in patients with chronic obstructive pulmonary disease. Thorax 2005; 60: 925-931.

89 Marin JM, Soriano JB, Carrizo SJ, et al. Outcomes in patients with chronic obstructive pulmonary disease and obstructive sleep apnoea: the overlap syndrome. Am J Respir Crit Care Med 2010; 182: 325-331.

90 Wertz DA, Pollack M, Rodgers K, et al. Impact of asthma control on sleep, attendance at work, normal activities, and disease burden. Ann Allergy Asthma Immunol 2010; 105: 118-123.

91 Sundberg R, Toren K, Franklin KA, et al. Asthma in men and women: treatment adherence, anxiety, and quality of sleep. Respir Med 2010; 104: 337-344.

92 Prescott E, Bjerg AM, Andersen PK, et al. Gender difference in smoking effects on lung function and risk of hospitalization for COPD: results from a Danish longitudinal population study. Eur Respir J 1997; 10: 822-827.

93 Lee KK, Birring SS. Cough and sleep. Lung 2010; 188: Suppl. 1, S91-S94.

94 Jarjour NN, Busse WW. Cytokines in bronchoalveolar lavage fluid of patients with nocturnal asthma. Am J Respir Crit Care Med 1995; 152: $1474-1477$.

95 Bates ME, Clayton M, Calhoun W, et al. Relationship of plasma epinephrine and circulating eosinophils to nocturnal asthma. Am J Respir Crit Care Med 1994; 149: 667-672.

96 Mackay TW, Wallace WA, Howie SE, et al. Role of inflammation in nocturnal asthma. Thorax 1994; 49: 257-262.

97 Kraft M, Djukanovic R, Wilson S, et al. Alveolar tissue inflammation in asthma. Am J Respir Crit Care Med 1996; 154: 1505-1510.

98 Panzer SE, Dodge AM, Kelly EA, et al. Circadian variation of sputum inflammatory cells in mild asthma. J Allergy Clin Immunol 2003; 111: 308-312.

99 Atanasov ST, Calhoun W. The relationship between sleep and asthma. Sleep Med Clin 2007; 2: 9-18. 